\title{
Baustein West und Bauplan Ost. Zur politischen Transformation jugendkultureller Stile in der DDR
}

\author{
Michael Rauhut
}

\section{Keywords}

Youth culture, German Democratic Republic, punks, hippies, protest, communist ideology, cultural policy.

\begin{abstract}
Within different political systems, youth cultures use similar symbols and styles which are charged with distinct and specific meaning. In the German Democratic Republic, the socialist part of Germany, youth cultures were identified as being a political threat. They created space for communication that was in sharp contrast to the propagandistic ideal of the so-called „socialist personality“ and hardly to control by the state. Based on the example of the hippie phenomenon in the East, the essay explains the way global youth cultures had been differentiated culturally and loaded with political sense under the circumstances of socialism.
\end{abstract}

Die Soziologen Manfred Stock und Philipp Mühlberg haben 1990 "Die Szene von innen” veröffentlicht, ein Buch über "Skinheads, Grufties, Heavy Metals und Punks" in der DDR. Vertreter dieser Szenen berichten hier über ihre Motivation und den jugendkulturellen Alltag unter realsozialistischen Bedingungen. Im Anhang verdichten die Herausgeber ihre Interviews zu einem theoretischen Resümee: Jugendkulturen bezogen im Osten ihre Inhalte maßgeblich aus dem gesellschaftlichen Konfliktpotenzial des Landes. Lediglich der Rohstoff, das stilistische Reservoir, stammte aus dem Westen. Oder wie es Stock und Mühlberg formulierten: “Die 'Baupläne' werden benutzt, um eigene Erfahrungen im Arrangement der Bausteine, in der Kombination von Zeichen manifest werden zu lassen.” (Stock 1990:236)

Ich möchte an diese These anknüpfen und untersuchen, auf welche spezifische Weise westliche Kulturmuster im Alltag von DDR-Jugendlichen reflektiert und politisch transformiert worden sind. Der Prozess und die Resultate der Umcodierung sollen anhand der ostdeutschen Hippiekultur exemplarisch dargestellt werden. 


\section{Rockmusik und Jugendkultur in der DDR}

Populäre Musik definiert sich in entscheidendem Maße über die kulturellen Gebrauchszusammenhänge, in die sie eingebunden ist. Von den Swingfanatikern der dreißiger Jahre über die Bewegungen der "Halbstarken", der Mods, Rocker und Hippies bis hin zu Punks, Gothics und Techno-Kids: Musik fungiert als Klammer über ein vielgestaltiges Reservoir von Symbolen, Verhaltensmustern und Attitüden, die zur Abgrenzung dienen. Nur der Kreis der Eingeweihten kann ihre Codes entschlüsseln. Ihr kultureller Kontext ist ein Raum der Selbstfindung und Selbstverwirklichung. Der britische Soziologe Simon Frith vermerkte zu Recht: "Der erste Grund, der diese Musik attraktiv macht, ist ihr Gebrauch zur Beantwortung von Fragen nach unserer Identität. Wir benutzen Popsongs, um uns eine bestimmte Art von Selbstdefinition zu schaffen, einen bestimmten Platz innerhalb der Gesellschaft. Das Vergnügen, das Popmusik produziert, ist ein Vergnügen der Identifikation - mit der Musik, mit den Künstlern, mit anderen, die sie ebenfalls mögen.” (Frith 1987:140)

Analog zu den Industrienationen der westlichen Hemisphäre und des Ostblocks war die Rock- und Popszene der DDR an die Batterie des angloamerikanischen Marktes angeschlossen. Von dort bezog sie musikalisch-stilistische Vorgaben und kulturelle Symbole. Vermittelt wurden die Sounds und Images durch die Medien der Bundesrepublik, aber auch Luxemburgs, Österreichs und der Alliierten. Nahezu sämtliche Trends des Westens fanden mit variierender Zeitverzögerung ihr ostdeutsches Imitat oder Pendant. Electronic Rock und die Disco-Welle hatten erst die technischen Hürden der Mangelwirtschaft zu überwinden, während der Punk lange Zeit von staatlicher Repression behindert wurde. Psychedelic Rock spielte im Live-Zusammenhang der DDR kaum eine Rolle, weil das konstituierende Moment kollektiver Drogenerfahrung fehlte. Handwerklich ambitionierte Spielweisen wie Art Rock und Classic Rock besaßen dagegen eine wesentlich höhere Lebensdauer als in ihren Ursprungsländern, was dem Kunstanspruch der Medien und der besonderen Qualifikation professioneller Musiker geschuldet war, die in der Regel ein Hochschulstudium zu absolvieren hatten. Beachtliche Resistenz zeigten Strömungen, die den Nimbus des “Handgemachten”, “Authentischen” trugen, wie etwa Folk Rock, Country Rock und Blues.

\section{Die politische Wahrnehmung von Jugendkulturen}

Die sozialen und kommunikativen Qualitäten des Rock wurden in der DDR durch das spezifische Klima der "geschlossenen Gesellschaft” aufgewertet. Diese Musik avancierte für 
viele zum Sinnbild für "Freiheit", "Widerstand" und "Anderssein". Unter ihrem Stern etablierten sich Nischen, Handlungsräume, in denen sonst offiziell verwehrte Erfahrungen gesammelt und Befindlichkeiten ausgelebt werden konnten. Mit den habituellen Eigentümlichkeiten und Attitüden der Fans, dem Gruppenverhalten der Gleichgesinnten oder den regelbrechenden Vorstellungen über Sexualität, Moral und Genuss wurde unablässig politischer Sprengstoff produziert. Ihn zu entschärfen und den Verlust an Boden zu stoppen, verschlang enorme Energien. Der Staat entwickelte ausgeklügelte Sicherheitsstrategien und initiierte Kampagnen, die die Rockkultur kontrollieren, zähmen und kanalisieren sollten. Was sich zu experimentell oder renitent gebärdete, wanderte in das Hoheitsgebiet privater Veranstalter und kirchlicher Einrichtungen ab.

Bis 1965 befasste sich die Spitze der SED mit den jugendkulturellen Phänomenen, wie sie im Zeichen der Beatmusik blühten - danach fiel die sicherheitspolitische Überwachung des Rockalltags komplett in das Ressort von Polizei und Stasi. Eine ministerielle "Dienstanweisung Nr. 4/66 zur politisch-operativen Bekämpfung der politischideologischen Diversion und Untergrundtätigkeit unter jugendlichen Personenkreisen in der DDR” vom 15. Mai 1966 definierte langfristige Strategien. Sie gab Interpretations- und Handlungsschablonen vor, die den Umgang der Stasi mit der einheimischen Rockkultur bis zum Fall der Mauer prägten. Dazu zählte die Manipulationsdoktrin, die in jeglicher Aufweichung des gesellschaftlichen Egalitätsprinzips eine "vom Gegner organisierte Feindtätigkeit" entdeckte: "Die Jugend der DDR stellt im System der psychologischen Kriegsführung einen besonderen Angriffspunkt dar. Ein koordiniertes Zusammenspiel zwischen dem Bonner Staatsapparat, den westlichen Geheimdiensten, den Agentenzentralen und Zentren der ideologischen Diversion, zwischen westdeutschen Jugendorganisationen, Film- und Starclubs, kirchlichen Institutionen, Rundfunk, Presse und Fernsehen u. a. ist darauf ausgerichtet, die Jugend der DDR vom Einfluss der sozialistischen Ideologie zu isolieren, in die Passivität zu drängen, eine Atmosphäre der allgemeinen Unsicherheit und zeitweilig in bestimmten Territorien Bedingungen zu schaffen, die zu Zusammenrottungen und Ausschreitungen Jugendlicher führen sollen.” (Dienstanweisung 1966:27) Die Dienstanweisung legte auch den strategischen Grundstein für die Observation und “Zersetzung” von "negativ-dekadenten Jugendlichen", wie das pauschalisierende Etikett fortan lautete. Sie schrieb vor: "Durch zielgerichtete Werbungen unter Mitgliedern der 
westlich orientierten Musikgruppen und ihrer Anhängerschaft ist eine ständige operative Kontrolle zu sichern.” (Dienstanweisung 1966:43)

Jugendkulturen wurden in der DDR nicht nur mit ästhetischen Ressentiments belegt und als Gefährdung von Ordnung und Sicherheit identifiziert, sondern zur politischen Bedrohung stilisiert. Dass Teens und Twens aus den vorgezeichneten Bahnen ausscherten und eigene Sozialisationsmuster entwickelten, dass sie autonome Kommunikationsräume besetzten, interpretierte der Staat als höchstbrisanten Angriff auf seine Autorität. Hier drohte außer Kontrolle zu geraten, was er eigentlich steuern wollte: die flächendeckende Verwirklichung des sozialistischen Persönlichkeitsideals auch jenseits der offiziellen Sphären von Schule und Beruf. Entsprechend allergisch fielen seine Reaktionen aus.

Die Auseinandersetzung mit einheimischen jugendkulturellen Phänomenen fand bis Ende der achtziger Jahre prinzipiell intern und unter ideologisch-verzerrten Vorzeichen statt; die Medien und die Propaganda richteten ihren Blick ausschließlich auf den Westen. Hippies, Punks oder Skinheads wurden als Krisensymptome des Kapitalismus wahrgenommen, als desillusionierte und manipulierte Jugendliche. Über die "Blumenkinder" war beispielsweise zu lesen: “Die Absage an ein sinnvolles Leben, die 'Kritik' an der Ausbeutergesellschaft durch die Flucht zur Droge und zum Narkotikum Musik, genau das ist die Lebenshaltung, die die zum Untergang verurteilte Gesellschaftsordnung braucht, um ihre Lebenszeit zu verlängern.” (Hofmann 1971:72)

\section{Hippies in der DDR: jugendkulturelle Ausdifferenzierung und politischer Sinn}

Ich möchte im Folgenden drei Thesen zur Ausdifferenzierung von Jugendkulturen in der DDR zur Diskussion stellen. Meine Illustrationen beschränken sich exemplarisch auf eine Szene, deren Repräsentanten als "Blueser", "Kunden” oder "Tramper" bezeichnet wurden und die als ostdeutsche Variation der Hippies gelten können.

Erstens: Jugendkulturen zeichneten sich in der DDR durch eine Erweiterung des stilistischen Repertoires der aus dem Westen transferierten Images und Verhaltenskodizes aus. Stellt man den "Blueser" Marke Ost und den Hippie aus San Francisco nebeneinander, fallen feine Unterschiede im Outfit auf. Beide tragen lange Haare und Bärte, Jeans und Sandalen, wallende Röcke, Stirnbänder, Ketten, Amulette und das obligatorische PeaceZeichen. Beim "Blueser” fällt der anhaltende Kult um den Parka der US-Army auf, der spätestens seit der Mod-Bewegung zu den Fetischen der Rockgeschichte gehört und der in der 
DDR zu horrenden Schwarzmarktpreisen gehandelt wird. Außerdem kombiniert der "Blueser" die Versatzstücke des westlichen Dresscodes mit landestypischen Gegenständen des täglichen Gebrauchs. Er trägt Arbeitshemden, Kletterschuhe, die eigentlich für Bergsteiger gedacht sind, verstaut seine Siebensachen am liebsten in einem altmodischen Hebammenköfferchen und treibt mit diesem Patchwork aus "originär" und "innovativ" das Prinzip der Bricolage auf die Spitze. Die hinzugefügten Stilelemente sind nicht nur billig und leicht verfügbar, sie passen auch nahtlos ins ästhetische Konzept und offenbaren kreative Lust.

Akzentverschiebungen waren genauso für die Musik typisch. Wie schon die Selbstbezeichnung der Szenegänger ahnen lässt, stand der Blues im Mittelpunkt des Interesses. Natürlich gehörte diese Musik auch zum Klangkosmos der Hippies; sie feierten Janis Joplin und die Doors und hatten mit Jimi Hendrix ihren Gitarrengott. Der zentrale Stellenwert des Blues und das Ausblenden des psychedelischen Elements, wie es international etwa durch die Grateful Dead oder Jefferson Airplane repräsentiert wurde, waren ein Spezifikum der DDR. Die fast schon religiöse Verklärung des Blues speiste sich aus zwei Quellen: Sie war ein Relikt euro-romantischer Interpretationsmuster und zugleich Ausdruck der jugendlichen Verweigerungshaltung. In die Musik der Afroamerikaner wurden Werte wie "Echtheit" und "reine Emotion" projiziert, die Unterdrückung des "Schwarzen" galt als leidensgeschichtliches Ahnenmuster der "Blueser".

Als ein weiteres Indiz für die graduelle Transformation der Hippiekultur sei die soziale Verankerung in Ostdeutschland erwähnt. Im Unterschied zur studentischen und intellektuellen Klientel des Westens, rekrutierten sich die "Blueser" vorrangig aus Lehrlingen und jungen Industriearbeitern. Ihr Ausstieg aus der DDR war keineswegs total; sie beugten sich der rechtlich verbrieften "Pflicht zur Arbeit" und standen montags bis freitags an der Werkbank, bevor sie an den Wochenenden in eine Parallelwelt flüchteten. Dann frönten sie dem Exzess, der Drogen nur in Form von Alkohol kannte.

Zweitens: Jugendkulturen besaßen in der DDR eine größere soziale Halbwertszeit. Sie fassten im ostdeutschen Alltag zwar prinzipiell später als etwa in der Bundesrepublik Fuß, zeichneten sich aber durch eine höhere Lebensdauer aus. Während im Westen die Hippiewelle oder der Punkboom längst den Gipfel überschritten hatten, blühten hier die Phänomene weiter. Die anachronistische Zählebigkeit war das Resultat biotopischer Verhältnisse: Jugendkulturen wurden im Osten nicht in industrielle Verwertungsketten 
eingetaktet und der Logik des Marktes unterworfen. Der britische Popmusikkritiker George Melly hat den für den Westen typischen Algorithmus auf eine Formel gebracht: "was als Revolte beginnt, endet als Stil - als Manierismus”. (Melly 1972:43) Dieses Gesetz galt in der DDR nicht, zumindest nicht in seiner kommerziellen Zwangsläufigkeit. Weder der Look der Hippies noch der Punks, Gothics oder Metalfreaks und die damit assoziierten Attitüden wurden als Geldquelle erkannt und somit ihrer rebellischen Pose beraubt. In der DDR gab es diese Vermarktungsmechanismen nicht. Die Kleidungsstücke und Accessoires, die den Stil einer Szene prägten, die als Symbole der Abgrenzung funktionierten, konnte man nicht im Laden kaufen; man musste sie sich unter oftmals hohem Energieaufwand quasi "erkämpfen". Sie behielten ihre kulturelle und politische Sprengkraft und wurden weitaus weniger schnell entwertet als in den westlichen Ursprungsländern.

Die Szene der "Blueser", die von der Hippiebewegung der Endsechziger inspiriert war, blieb bis zur "Wende" von 1989 eine feste Größe des jugendkulturellen Alltags. Der ideologische Kanon der Flower Power - die tabufreien Auffassungen von Sexualität, Genuss und Moral, die Sehnsucht nach Freiheit und Individualität - behielt als Gegenentwurf zu Spießigkeit und Enge in der DDR eine geradezu magische Anziehungskraft. Und natürlich bezog die Szene einen Großteil ihrer Motivation auch aus dem Mythos vom Amerika der “unbegrenzten Möglichkeiten”, der zu den Konstanten jugendlicher Sehnsucht im Osten Deutschlands zählte.

Drittens: Der spezifische Sinn von Jugendkulturen war in der DDR politisch determiniert. Zwar lieferten die westlichen Vorbilder auch die ideologischen Fixpunkte - sie wurden aber von den gesellschaftlichen Konfliktfeldern absorbiert und fanden sich in neuen Bedeutungszusammenhängen wieder. Der Prozess ging mehrstufig vonstatten. Wer in der DDR "Blueser" oder Punk wurde, entschied sich gegen die politische Norm, gegen das sozialistische Erziehungsideal und Persönlichkeitsbild. Das zog automatisch den Vorwurf der "Staatsfeindlichkeit" und Sanktionen nach sich, die im Extremfall im Gefängnis endeten. Die Stigmatisierung erzeugte Gegenwehr in Gestalt einer zunehmend politischen Selbstverortung der Szenen. Ein Paradebeispiel lieferte die Geschichte des Punk in Ostdeutschland. Erst als sich um 1982/83 die staatlichen Repressalien zum Terror steigerten und Punks als Neofaschisten diffamiert wurden, bekam die Bewegung einen ausgesprochen politischen Akzent. Punks provozierten das System mit subversiven Songs und Aktionen, sie schlossen Kontakt zu oppositionellen Kreisen und bevölkerten den Schutzraum der Evangelischen 
Kirche. Geprägt durch die speziellen sozialen Erfahrungen, unterschied sich ihr Weltbild gravierend von den No-Future-Sprüchen der Abrisshausguerilla im Westberliner Kreuzberg.

Die letzte Stufe in der Auseinandersetzung zwischen Macht und Szene bildete die Strategie der kulturpolitischen Vereinnahmung. Sie erwies sich als der wirkungsvollste Weg der Paralyse und hatte unterm Strich den gleichen Effekt wie die Kommerzialisierung im Westen: Jugendkulturen mutierten zum Gemeingut, zur amtlichen Form von Unterhaltung und büßten ihre polarisierende Kraft ein. Punkbands, die noch Anfang der Achtziger verboten waren, wurden ein halbes Jahrzehnt später mit staatlichen Subventionen, Plattenverträgen und medialem Beifall geködert. Das Phänomen verlor seinen Stachel.

Auch die Kultur der "Blueser" trug die Spuren der politischen Aufladung nach dem Schneeballprinzip. Die langhaarigen Jeans- und Parkaträger standen von Anfang an im Fadenkreuz der Sicherheitsorgane. Sie wurden als "politisch-negativ", "westlich-dekadent" und "asozial" abgestempelt. Analog zu ihrer Verbreitung wuchs der Druck von Polizei und Stasi. Als im letzten Drittel der siebziger Jahre die Bewegung den Zenit erreichte, spitzte sich die Repression zu. Geheimdienstchef Erich Mielke befahl am 14. Januar 1978 die "politischoperative Arbeit" landesweit zu verstärken und im Ernstfall "das sozialistische Strafrecht konsequent anzuwenden”. (Dienstanweisung 1978:o.p.) Stück für Stück verfeinerte das MfS sein strategisches Repertoire und perfektionierte das Spitzelsystem. Dienstanweisungen, Schulungen und Graduierungsschriften der Juristischen Hochschule Potsdam lieferten detaillierte Analysen und Pläne. "Operative Vorgänge” und "Operative Personenkontrollen” mit Decknamen wie "Blues", "Penner", “Tramper", “Anhalter" oder "Diestel” richteten das Visier auf besonders suspekte "Langhaarige" und Musiker. Sie wurden nicht selten über Jahre hinweg observiert, in ihrem Wirkungsfeld eingeschränkt und durch subtilen Terror langsam gelähmt - oder wie es die Stasi nannte: "zersetzt".

Die Methoden bündelte der “Operative Vorgang Tramper”, im Mai 1978 von der Bezirksverwaltung Gera eingeleitet. Ihre Aktion richtete sich gegen eine 40 bis 50 Personen umfassende, im Kern 15 Mann starke Gruppe "politisch und moralisch labiler Jugendlicher". Der Plan sah vor, sie "umsichtig, vielschichtig, differenziert und schnell unter Einbeziehung aller geeigneten Erziehungsträger zurückzudrängen, zu differenzieren, zu verunsichern, in ihrer 'Freizügigkeit' einzuengen und letztendlich aufzulösen”. (Bezirksverwaltung 1978:19, 25) 1982 resümierte man den "Zerfall und die Zersetzung der Gruppierung" (Bezirksverwaltung 1982:323), das Dossier wurde geschlossen. Die Stasi und ihre vier 
geschickt platzierten Inoffiziellen Mitarbeiter hatten ganze Arbeit geleistet: Fünf Mitglieder der Tramper-Clique gingen ins Gefängnis, einer in den Westen, ein anderer wurde zum Wehrdienst einberufen und damit "unschädlich gemacht".

Im Kern eine hedonistische Jugendkultur, vermischten sich die Randbereiche der "Blueser"-Szene zusehends mit oppositionellen Strömungen. Die "Offene Arbeit" der Evangelischen Kirche, die eine konfessionslose Klientel ansprach, wurde zum Sammelbecken unangepasster Jugendlicher. Etliche von ihnen definierten sich selbst als "Blueser". Der prominenteste Fall war Matthias Domaschk aus Jena, 1957 geboren und am 12. April 1981 unter ungeklärten Umständen in Stasihaft gestorben. Auf Initiative des Thüringer Pfarrers Walter Schilling fanden 1978 und 79 in Rudolstadt die überregionalen Begegnungswerkstätten "June" statt, die mit Ausstellungen, Lesungen, Gottesdiensten und Konzerten vor allem langhaarige Querdenker anzogen. Zwischen 1979 und 1986 richteten sich die Ostberliner "Bluesmessen" direkt an diese Zielgruppe; später fand sie in der kirchlichen Friedens- und Ökobewegung eine Heimstatt.

Die staatliche Vereinnahmung, die letzte Phase der Politisierungsspirale, blieb den "Bluesern" übrigens erspart. Ab Mitte der Achtziger litten sie zunehmend unter Nachwuchsproblemen, weil neue Jugendkulturen attraktiver wurden. Nun ballten grellschopfige Punks die Faust gegen das System, schockten Heavy-Metal-Fans mit Muskeln und Leder und widerlegte eine wachsende Schar von Skinheads auf wahrhaft schlagende Weise, dass es in der DDR keinen Neonazismus gebe. Die Sicherheitsorgane sahen sich mit anderen, größeren Problemen konfrontiert. Es war nur noch eine Frage der Zeit, bis das Schreckgespenst des "Bluesers" von der politischen Agenda verschwand.

\section{Literatur:}

Bezirksverwaltung für Staatssicherheit Gera, Abteilung XX/2 (1978). Sachstandsbericht zu einer negativ-dekadenten jugendlichen Gruppierung aus Gera, 5. Mai 1978. Die Bundesbeauftragte für die Unterlagen des Staatssicherheitsdienstes der ehemaligen Deutschen Demokratischen Republik (BStU), MfS BV Gera, AOP 924/82.

Bezirksverwaltung für Staatssicherheit, Kreisdienststelle Gera (1982). Abschlussbericht zum OV “Tramper" - Reg.-Nr. 332/78, 20. September 1982. BStU, MfS BV Gera, AOP 924/82. 
Dienstanweisung Nr. 4/66 zur politisch-operativen Bekämpfung der politisch-ideologischen Diversion und Untergrundtätigkeit unter jugendlichen Personenkreisen in der DDR, 15. Mai 1966. BStU, Zentralarchiv (ZA), MfS VVS 008-365/66.

Dienstanweisung Nr. 8/78, 14. Januar 1978. BStU, ZA, VVS MfS 008-8/78, Dok. 102433.

Frith, S. (1987). Towards an Aesthetic of Popular Music. In: R. Leppert \& S. McClary (Ed.), Music and Society. The Politics of Composition, Performance and Reception (pp.133-149). Cambridge: Cambridge University Press.

Hofmann, H. P. (1971). ABC der Tanzmusik. Berlin: Verlag Neue Musik.

Melly, G. (1972). Revolt into Style. The Pop Arts in Britain. London: Penguin Books.

Stock, M. \& Mühlberg, P. (1990). Die Szene von Innen. Skinheads, Grufties, Heavy Metals, Punks. Berlin: LinksDruck. 